\title{
Retraction of: Tumor Protein D52-Like 2 Accelerates Gastric Cancer Cell Proliferation; 10.1089/cbr.2014.1766
}

Cancer Biotherapy and Radiopharmaceuticals $(C B R)$ is officially retracting the published article by Jiapeng Xu, Weimin Wang, Zhenxin Zhu, Ziran Wei, Dejun Yang, and Qingping Cai entitled, "Tumor Protein D52-Like 2 Accelerates Gastric Cancer Cell Proliferation In Vitro," Cancer Biother Radiopharm June 2015, 30(3): 111-116; DOI: 10.1089/cbr.2014.1766

The editors of CBR received a letter from an investigator raising concerns regarding this published paper. The investigator claims that this study employed the 5'-GTAG.... shRNA sequence as a "negative control," despite the fact that according to Blastn analyses, this sequence shows homology to the human NOB1 gene. The investigator's concern is that since the article did not employ a non-targeting lentiviral construct as described in the study, it most likely invalidates the experimental results reported in the article. The authors of the article were afforded the opportunity to respond, and they disagreed with the concerns. However, the editors have decided to retract the article based on our published understanding of the incorrect use of nucleotide sequence reagents by this publication and the serious nature of the plausibility of the raised concerns.

It is important to note that an earlier e-pub version of this retraction notice was posted with unintended errors and have been corrected within this official retraction notice. A list of the affected text appears below.

Cancer Biotherapy and Radiopharmaceuticals is dedicated to upholding the strictest standards of peer review and the scientific record and therefore is retracting this article.

\section{List of corrected text from e-published versions of this retraction:}

Original retraction text: ... this study employed the 5'-GCGG... shRNA sequence as a "TPD52L2 targeting sequence", despite the fact that this sequence shows no homology with any TPD52L2 sequence, or indeed any sequence in the nonredundant nucleotide database.

- CORRECTED text: ... this study employed the 5'-GTAG.... shRNA sequence as a "negative control," despite the fact that according to Blastn analyses, this sequence shows homology to the human NOB1 gene.

Original retraction text: ... since the article did not employ a lentiviral construct targeting the TPD52L2 gene as described in the study, it most likely invalidates the experimental results reported in the article.

- CORRECTED text: ... since the article did not employ a non-targeting lentiviral construct as described in the study, it most likely invalidates the experimental results reported in the article. 